\title{
Programa de oxigenoterapia domiciliar para crianças egressas de uma unidade neonatal: relato da experiência de dez anos
}

\author{
Home oxygen therapy program for infants after neonatal unit discharge: report of a ten-year experience
}

\author{
Elaine Aparecida L. Garcia', Maria Aparecida Mezzacappa², Mônica Aparecida Pessoto ${ }^{3}$
}

\section{RESUMO}

Objetivo: Descrever os resultados do programa de oxigenoterapia domiciliar, com uso de concentrador de oxigênio, utilizado por crianças egressas de uma unidade neonatal ao longo de seus primeiros dez anos.

Métodos: Estudo de corte transversal com componente analítico para avaliar o programa de oxigenoterapia domiciliar de uma unidade neonatal de um hospital público universitário do interior do Estado de São Paulo durante o período de novembro de 1996 a dezembro de 2006.

Resultados: Foram identificadas 160 crianças com indicação de oxigenoterapia domiciliar, das quais $63,3 \%$ apresentavam displasia broncopulmonar. Em média, 3,0\% de todos os pacientes internados e 8,6\% dos recém-nascidos com peso inferior a $1500 \mathrm{~g}$ ao nascer receberam alta em oxigenoterapia. A duração média do uso de $\mathrm{O}_{2}$ no domicílio foi de 42,3 $\pm 54,0$ dias. Na comparação das características demográficas da população e do tempo de uso de $\mathrm{O}_{2}$ domiciliar, não se observaram diferenças significativas entre os anos. Não houve correlação entre o tempo de ventilação mecânica e a duração da terapia domiciliar. Houve necessidade de cuidados especiais, além do uso de $\mathrm{O}_{2}$ e medicações, em 22,3\% dos casos. Nos primeiros dois anos de vida, a taxa de morbidade e de mortalidade foram, respectivamente, 40,1 e 14,1\%.

Conclusões: $\mathrm{O}$ uso de oxigênio domiciliar, com sistema de concentrador de oxigênio, é uma alternativa terapêutica factível em nosso meio, merecendo mais atenção dos profissionais de saúde e maior investimento das instituições de saúde. São necessários mais estudos nacionais para se

Instituição: Departamento de Pediatria da Faculdade de Ciências Médicas da Universidade Estadual de Campinas (Unicamp); Hospital da Mulher Prof. Dr. José Aristodemo Pinotti - Centro de Atenção Integral à Saúde da Mulher (Caism), Campinas, SP, Brasil

${ }^{1}$ Mestre em Saúde da Criança e do Adolescente; Enfermeira Assistencial do Serviço de Neonatologia do Caism da Unicamp, Campinas, SP, Brasil ²Doutora em Saúde da Criança e do Adolescente; Professora do Departamento de Pediatria da Faculdade de Ciências Médicas da Unicamp, Campinas, SP, Brasil

${ }^{3}$ Doutora em Saúde da Criança e do Adolescente; Médica Assistente do Setor de Neonatologia da Unicamp, Campinas, SP, Brasil aprimorar a qualidade do atendimento e a segurança destes programas para a população neonatal.

Palavras-chave: oxigenoterapia; displasia broncopulmonar; prematuro; neonatologia; assistência domiciliar.

\section{ABSTRACT}

Objective: To describe the results of a home oxygen therapy program consisting of the use oxygen concentrator by children discharged from a neonatal unit during their first 10 years of the program.

Methods: Cross-sectional analytical study with data collection from November 1996 to December 2006. All infants that needed home oxygen therapy after discharge from the neonatal unit of a public university hospital in the state of São Paulo, Brazil, were studied.

Results: We identified 160 infants who used home oxygen therapy during the period and $63.3 \%$ of them had bronchopulmonary dysplasia. A mean of $3.0 \%$ of all hospitalized patients and $8.6 \%$ of very low birth weight newborns were discharged on oxygen. The mean duration of home oxygen therapy was $42.3 \pm 54.0$ days. Significant differences were not found when comparing demographic characteristics of the population and duration of home oxygen therapy. There was no correlation between the duration of mechanical ventilation and the duration of home oxygen therapy. In addition to the use of oxygen and medications, $22.3 \%$ of the infants needed special care. In the first 2 years of life, the infants' morbidity and mortality rates were, respectively, $40.1 \%$ and $14.1 \%$.

Endereço para correspondência:

Elaine Aparecida L. Garcia

Rua Hermantino Coelho, 195, apto. 123

CEP 13087-500 - Campinas/SP

E-mail: nanigarcia5@hotmail.com

Conflito de interesse: nada a declarar

Recebido em: 21/7/09

Aceito em: 1/12/09 
Conclusions: The use of an oxygen concentrator at home is a feasible therapeutic alternative in Brazil; therefore, it deserves more attention from health professionals and greater investments from health institutions. Further national studies are needed to improve the quality of care and the safety of these programs intended for the neonatal population.

Key-words: oxygen therapy; bronchopulmonary dysplasia; premature infant; neonatology; home nursing.

\section{Introdução}

A oxigenoterapia domiciliar é utilizada há aproximadamente 50 anos. Contudo, só a partir da década de 1970 foi confirmado que tal terapêutica promove a qualidade e prolonga a expectativa de vida de adultos com doença pulmonar obstrutiva crônica ${ }^{(1)}$.

Devido aos avanços tecnológicos no tratamento de recémnascidos (RN) muito prematuros, a displasia broncopulmonar (DBP) passou a figurar como causa de internação prolongada pela dependência crônica de oxigênio $\left(\mathrm{O}_{2}\right)^{(2,3)}$. O aumento do tempo de internação é acompanhado do risco de infecções hospitalares, separação familiar e altos custos para as instituições de saúde ${ }^{(3,4)}$. Neste contexto, a implantação de programas de oxigenoterapia domiciliar tem importante implicação médica, econômica e social.

Programas de oxigenoterapia domiciliar em crianças com DBP têm sido utilizados há cerca de $40 \operatorname{anos}^{(5)}$. O suplemento de $\mathrm{O}_{2}$ através de cateteres nasais mantém os níveis de pressão parcial de $\mathrm{O}_{2}$ adequados, promovendo redução tanto da resistência vascular pulmonar ${ }^{(6)}$ como da resistência das vias aéreas superiores ${ }^{(7)}$, reduzindo o risco de morte súbita ${ }^{(8)}$ e favorecendo o ganho ponderal ${ }^{(9,10)}$.

$\mathrm{Na}$ América Latina, poucos estudos sobre oxigenoterapia domiciliar em neonatos foram publicados. O Chile passou a utilizar esta modalidade terapêutica em 1990 e, desde 2003, dispõe de um programa nacional de oxigenoterapia domiciliar $^{(2)}$. No Brasil, não foram encontrados relatos sobre o tema em recém-nascidos e são raros os estudos com crianças ${ }^{(4)}$. Segundo a Rede Brasileira de Pesquisas Neonatais, no período de janeiro de 2006 a junho de 2008, apenas $3 \%$ dos RN com peso de nascimento entre 500 e $1500 \mathrm{~g}$ de oito instituições do sul e sudeste utilizaram $\mathrm{O}_{2}$ domiciliar ${ }^{(11)}$.

$\mathrm{O}$ uso domiciliar do $\mathrm{O}_{2}$, quando bem indicado, não aumenta o risco de reinternações nem promove elevação nos custos, mesmo considerando os gastos com o seguimento ambulatorial $^{(12)}$. Tendo em conta esta economia de recursos hospitalares ${ }^{(4,12)}$, a otimização do uso de leitos e as dificuldades de acesso e o financiamento da assistência à saúde neonatal no país, é pertinente descrever os dez anos de experiência de um programa de oxigenoterapia domiciliar com concentradores de $\mathrm{O}_{2}$ utilizado por crianças egressas de uma unidade neonatal brasileira e analisar a tendência desta modalidade terapêutica ao longo deste período.

\section{Método}

Foi realizado um estudo observacional, de coorte transversal, com componente analítico, para descrever a prevalência de uso e as características da população atendida por um programa de oxigenoterapia domiciliar com concentradores de $\mathrm{O}_{2}$ utilizado por crianças egressas da unidade neonatal de um hospital público universitário do Estado de São Paulo, no período de novembro de 1996 a dezembro de 2006. Foi analisada a tendência desta modalidade terapêutica ao longo de dez anos.

Foram incluídos no estudo todos os casos identificados no período cujos prontuários puderam ser acessados e que receberam alta hospitalar e se mantiveram em oxigenoterapia domiciliar. $\mathrm{O}$ critério para inclusão no programa de $\mathrm{O}_{2}$ domiciliar foi a manutenção da necessidade clínica de $\mathrm{O}_{2}$, constatada após várias tentativas de suspensão, em RN com condições clínicas e sociais para alta hospitalar.

Durante todo o período de estudo, foram utilizados os requisitos recomendados pela Academia Americana de Pediatria (AAP) (1998) para a alta ${ }^{(13)}$ : estabilidade clínica, necessidade de concentrações baixas de $\mathrm{O}_{2}$, com fluxo máximo aceitável de $1 \mathrm{~L} /$ min por cateter nasal para manter a $\mathrm{SaO}_{2}$ por oximetria de pulso entre 90 e $94 \%^{(14)}$. Para os pacientes com cardiopatias, a avaliação quanto aos níveis adequados de $\mathrm{SaO}_{2}$ foi individualizada. A instituição adotou ainda como critérios para a alta a ausência de apneias há pelo menos 48 horas, curva ascendente de peso e peso no momento da alta superior a $2000 \mathrm{~g}$, além de condições familiares e domiciliares adequadas.

Após a concordância da família com a alta em oxigenoterapia, o serviço social averiguou caso a caso os requisitos necessários para o pleno funcionamento do programa, como: disponibilidade de espaço físico, rede de energia elétrica, condições para pagamento da conta de luz, proximidade entre o domicílio e a unidade básica de saúde e disponibilidade de transporte para os retornos de rotina e 
urgência. Concomitantemente, a unidade básica de saúde foi comunicada para promover facilidades de transporte para a família. O passo seguinte foi a locação, pela instituição hospitalar, de um torpedo de $\mathrm{O}_{2}$ e um aparelho concentrador de $\mathrm{O}_{2}$, fornecidos aos familiares mediante empréstimo. Oxímetros de pulso não foram utilizados no domicílio por estes pacientes.

O concentrador de $\mathrm{O}_{2}\left(\right.$ Air Liquide $\left.{ }^{\circledR}\right)$ é um equipamento alimentado por energia elétrica e constituído por filtro molecular que extrai o nitrogênio e outros gases do ar ambiente e os enriquece com oxigênio até concentrações entre 90$95 \%$, dependendo do fluxo que varia de 0,25-5L/min. Este equipamento permite certo grau de mobilidade ao paciente, já que é provido de rodízios, tem menor risco de explosão e prescinde de recarga ${ }^{(1,15)}$.

Antes da alta hospitalar, foi feito o treinamento das mães para a utilização do sistema (concentrador/torpedo) por meio da internação do binômio em alojamento conjunto. Neste local, a mãe recebeu informações sobre a manipulação do torpedo e concentrador de $\mathrm{O}_{2}$, sobre a alteração do fluxo de $\mathrm{O}_{2}$ de 0,25 a $5 \mathrm{~L} / \mathrm{mim}$, em situações de cianose/aumento do esforço respiratório, bem como sobre os procedimentos em situações de urgência e telefones da assistência técnica para os equipamentos. Foram também transmitidas informações sobre cuidados gerais, alimentação e medicações.

Após a alta, realizaram-se retornos ambulatoriais, inicialmente semanais, para avaliar a possibilidade de suspensão intermitente ou total do $\mathrm{O}_{2}$ e acompanhamento geral da criança. Nos retornos, $\mathrm{o}_{2}$ foi suspenso e as crianças monitoradas com oximetria de pulso por várias horas, sendo a $\mathrm{SaO}_{2}$ observada durante o sono ${ }^{(16)}$, alimentação ${ }^{(17)} \mathrm{e}$ em estado de alerta ${ }^{(16)}$. Os limites de oximetria de pulso utilizados foram os mesmos da internação ${ }^{(14)}$. Após a suspensão do $\mathrm{O}_{2}$, os neonatos foram acompanhados até dois anos de idade.

As variáveis demográficas avaliadas foram: peso ao nascer, gênero, idade gestacional pelo método de Capurro ${ }^{(18)}$ ou New Ballard $^{(19)}$, doença de base associada à dependência de $\mathrm{O}_{2}$, tempo de oxigenoterapia durante a internação, duração da internação, peso e idade pós-menstrual (idade gestacional acrescida do tempo de vida pós-natal em semanas) à alta hospitalar, tempo de treinamento dos pais, número de retornos ambulatoriais e de reinternações hospitalares, causas de óbito, idade corrigida à suspensão do $\mathrm{O}_{2}$ domiciliar e duração da oxigenoterapia domiciliar em dias, condições socioeconômicas e grau de instrução da família. Avaliou-se, ainda: alimentação, medicações, dispositivos ou procedimentos (sondas, traqueostomias, gastrostomias, aspiração de vias aéreas superiores) utilizados pelo $\mathrm{RN}$ à alta e intercorrências no uso do concentrador (incêndios, quebra ou danos ao equipamento que tenha levado à descontinuação do $\mathrm{O}_{2}$ ).

Para a análise estatística, foi utilizado o SAS for Windows (Statistical Analysis System), versão 9.1.3 Service Pack 3 (SAS Institute Inc, 2002-2003, Cary, NC, USA). Realizou-se a análise descritiva com cálculo das médias, medianas, desvio padrão e valores mínimos e máximos. A tendência nas frequências de uso do $\mathrm{O}_{2}$ por ano foi verificada através do teste de Cochran-Armitage. Para verificar a associação linear entre a duração da oxigenoterapia na internação hospitalar e no domicílio para os casos com DBP, utilizou-se o coeficiente de correlação de Spearman. A fim de se comparar as medidas contínuas ao longo dos anos, foi utilizado teste ANOVA com transformação por postos. O nível de significância adotado para os testes estatísticos foi de 0,05. O estudo foi aprovado pelo Comitê de Ética em Pesquisa da instituição.

\section{Resultados}

Foram identificados 160 recém-nascidos que receberam alta hospitalar com oxigênio domiciliar. Por dificuldade de acesso aos prontuários, não foi possível obter informações referentes a dois casos. Do total de casos, 128 (81\%) eram prematuros e $80(50,6 \%)$ masculinos. A descrição da população estudada, a duração da internação e do uso de oxigênio intra-hospitalar e no domicílio, bem como a idade corrigida à alta hospitalar e à suspensão do $\mathrm{O}_{2}$, são apresentadas na $\mathrm{Ta}$ bela 1. A duração da oxigenoterapia em casa foi estabelecida para 131 pacientes.

As principais doenças associadas à dependência crônica de oxigênio estão apresentadas na Tabela 2. A média $\pm D P$ do peso ao nascer e idade gestacional dos $\mathrm{RN}$ com DBP foi respectivamente de $1047,5 \pm 414,4 \mathrm{~g}$ e $29,7 \pm 2,8$ semanas. Durante a internação, o $\mathrm{O}_{2}$ foi administrado através de tendas, halo e cateteres nasais. Além de oxigênio inalatório, 127 casos (83\%) utilizaram ventilação mecânica invasiva. A média $\pm D P$ para esta variável foi de 20,2 $\pm 19,6$ dias, variando de 1 a 86 dias. Quanto ao uso de pressão positiva contínua nas vias aéreas (CPAP), 88 casos (58\%) utilizaram esta modalidade, com duração média de 5,5 55,3 dias. Nos RN com BDP, não houve correlação significativa entre o tempo total de utilização do $\mathrm{O}_{2}$ e a duração da ventilação mecânica durante a internação com o tempo de uso do $\mathrm{O}_{2}$ domiciliar.

Observou-se que dez RN (7,6\%) utilizaram oxigênio no ambiente domiciliar por menos de uma semana, sendo 
Tabela 1 - Peso ao nascer e à alta, idade gestacional e idade pós-menstrual à alta, tempo total de uso de oxigênio na internação, duração da internação e duração total do oxigênio domiciliar $(n=158)$

\begin{tabular}{lrccc}
\hline Variável & Média & Mediana & DP & Variação \\
\hline Peso ao nascer (gramas) & 1554,0 & 1675,0 & 898,4 & $510-4040$ \\
Idade gestacional (semanas) & 32,0 & 31,9 & 4,3 & $25-42$ \\
$\mathrm{O}_{2}$ internação (dias) & 73,3 & 70,0 & 39,1 & $10-260$ \\
Internação (dias) & 74,2 & 69,0 & 39,7 & $12-257$ \\
Peso à alta (gramas) & 2633,0 & 2450,0 & 579,4 & $1890-4620$ \\
IPM à alta (semanas) & 42,5 & 41,3 & 5,2 & $35-64$ \\
$\mathrm{O}_{2}$ domiciliar (dias) & 42,3 & 22 & 54,0 & $2-347$ \\
\hline
\end{tabular}

IPM: idade pós-menstrual; DP: desvio padrão; *Informação para 131 casos.

Tabela 2 - Distribuição absoluta e relativa dos diagnósticos associados à indicação de $\mathrm{O}_{2}$ domiciliar $(n=155)$

\begin{tabular}{lrr}
\hline Doenças $^{*}$ & \multicolumn{1}{c}{ n } & \multicolumn{1}{c}{$\%$} \\
\hline Displasia broncopulmonar & 100 & 64,5 \\
Doença do refluxo gastroesofágico & 49 & 32,0 \\
Cardiopatias & 12 & 7,7 \\
Sequelas de síndrome hipóxico-isquêmica & 12 & 7,7 \\
Restrição da caixa torácica & 7 & 4,5 \\
Síndromes genéticas & 7 & 4,5 \\
Mielomeningocele toracolombar & 5 & 3,2 \\
Sequelas de pneumonia & 3 & 1,5 \\
Hipoplasia pulmonar & 2 & 1,3 \\
\hline
\end{tabular}

*39 casos com mais de um diagnóstico. Informações não disponíveis para três casos.

que três mantiveram a terapia por apenas três dias. Dez casos permaneceram mais de 150 dias em oxigenoterapia domiciliar, dos quais três tinham cardiopatias, quatro DBP associada à doença do refluxo gastroesofágico (DRGE) e três apresentavam apenas DBP.

Quanto à distribuição do número de casos que utilizaram oxigenoterapia domiciliar por ano, em relação ao total de internados e em relação ao total de $\mathrm{RN}$ com peso ao nascer abaixo de $1500 \mathrm{~g}$, não ocorreram diferenças significativas ao longo dos anos (Figura 1). A média da frequência de $\mathrm{O}_{2}$ domiciliar em relação ao total de $\mathrm{RN}$ internados e o de $\mathrm{RN}$ com peso $<1500 \mathrm{~g}$ foi, respectivamente, 3,0 e 8,6\%.

Não foram constatadas diferenças significativas na comparação entre os anos do peso ao nascer, idade gestacional, duração da internação, idade corrigida à alta e à suspensão do $\mathrm{O}_{2}$. A duração de uso do $\mathrm{O}_{2}$ domiciliar não variou significativamente no decorrer dos dez anos do programa (Figura 2).

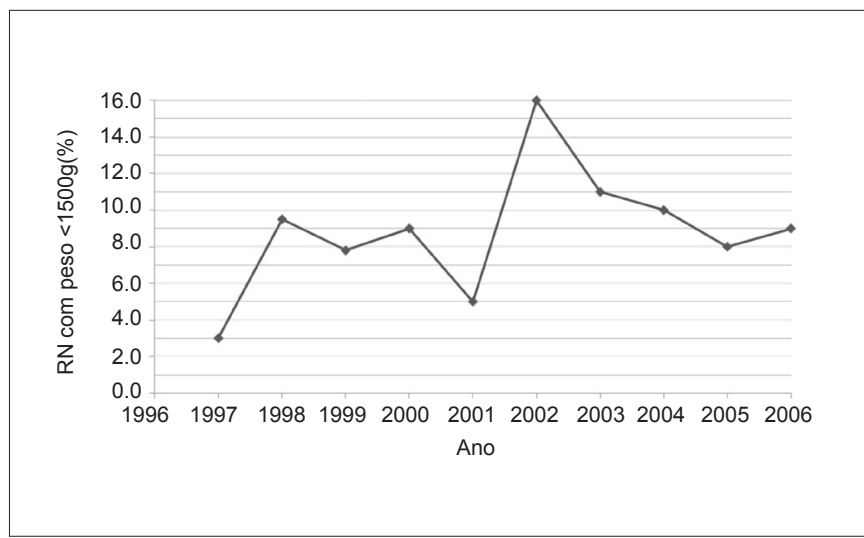

Figura 1 - Distribuição percentual do número de casos com peso ao nascer $<1500 \mathrm{~g}$ em $\mathrm{O}_{2}$ domiciliar em relação ao total de crianças nascidas no período nesta faixa de peso (teste de Cochran-Armitage; $p=0,107)$.

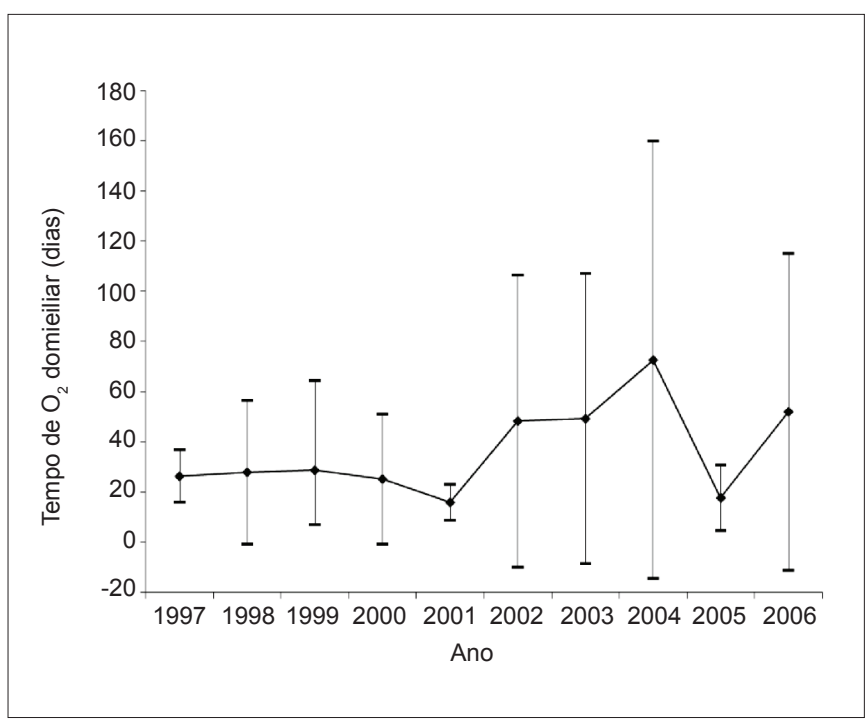

Figura 2 - Valores médios e desvios padrão da duração total, por ano, da oxigenoterapia domiciliar no período de novembro de 1997 a dezembro de 2006 ( $n=131$; ANOVA: $p=0,375)$. 
Em relação aos cuidados prestados aos pacientes no domicílio, a média $\pm D P$ do número de doses de medicações administradas pelos cuidadores foi 4,6 63,5 , variando de 1 a 16 doses diárias. Um total de $31 \mathrm{RN}(22,3 \%)$ tiveram alta hospitalar utilizando sondas enterais, seis $(4,3 \%)$ usando sondas de gastrostomia e três $(2,2 \%)$ de traqueostomia. $\mathrm{O}$ período de treinamento e orientação das mães para alta foi de 1,3 $\pm 0,7$ dias (variando de um a seis dias).

Só foi possível estudar as condições socioeconômicas e o grau de instrução em uma pequena subamostra da população $(\mathrm{n}=20)$, no período de 2003 a 2006. A renda per capita dos familiares foi de $\mathrm{R} \$ 189 \pm 88$, variando de $\mathrm{R} \$ 80$ a 387 . Completaram apenas o ensino fundamental e o médio, respectivamente, 5 e $45 \%$ das mães. Tiveram ensino fundamental e médio incompletos, respectivamente, 40 e $10 \%$ dos casos.

Não foram encontrados registros de possíveis intercorrências no domicílio com os concentradores de $\mathrm{O}_{2}$.

Foram registrados dados sobre o seguimento ambulatorial de $127 \mathrm{RN}$ em oxigenoterapia domiciliar, dos quais 114 acompanhados durante os primeiros dois anos de vida. Cinquenta e um casos $(40,1 \%)$ tiveram no mínimo uma reinternação hospitalar devido à doença pulmonar. Constatou-se que 18 pacientes $(14,1 \%)$ evoluíram a óbito neste período, sendo que oito deles $(6,2 \%)$ ainda utilizavam oxigênio domiciliar quando sofreram agudização da doença e foram re-hospitalizados, falecendo por insuficiência respiratória.

\section{Discussão}

O programa de oxigenoterapia domiciliar, implantado no serviço a partir de 1996, foi utilizado em média por 3,0\% dos pacientes internados e por $8,6 \%$ dos $\mathrm{RN}$ com peso inferior a $1500 \mathrm{~g}$, sendo a displasia broncopulmonar a doença mais prevalente entre estes recém-nascidos.

Esperava-se que a experiência adquirida no decorrer destes dez anos, somada aos avanços técnicos e científicos, implicaria três mudanças no perfil demográfico desta população: a redução da idade gestacional, do peso ao nascer e da idade corrigida no momento da alta. Todavia, tanto as características demográficas como a frequência de uso de $\mathrm{O}_{2}$ não variaram durante o estudo. Esse fato pode estar associado à manutenção dos critérios de alta neste período, principalmente em relação aos parâmetros de saturação de $\mathrm{O}_{2}$ utilizados pelo serviço, peso à alta a partir de $2000 \mathrm{~g}$ e à condição social dos familiares. Também colaborou para estes resultados o alto grau de dependência e gravidade dos pacientes, o que pode ter dificultado a alta mais precoce.
Em estudos sobre oxigenoterapia domiciliar realizados na última década, a população atendida apresentou média de idade gestacional de 25,2 a 28,5 semanas ${ }^{(20,21)}$ e peso ao nascer de 737 a $1106 \mathrm{~g}^{(20,22)}$, ambos valores inferiores aos obtidos neste estudo. Entretanto, a idade corrigida à alta hospitalar foi superior ou inferior à deste estudo ${ }^{(21,23)}$, o que pode denotar diferenças quanto aos protocolos de alta. Outro aspecto que justifica tais diferenças é a inclusão, neste estudo, de todos os neonatos que necessitaram de $\mathrm{O}_{2}$ e não apenas daqueles com displasia broncopulmonar.

Quanto à duração do $\mathrm{O}_{2}$ domiciliar, a média de uso em outros serviços variou de 63 a 97 dias $^{(8,21)}$ e, neste estudo, foi de 42,3 $\pm 54,0$ dias. Supõe-se que este resultado está associado às diferenças nos limites de $\mathrm{SaO}_{2}$ utilizados nos vários centros ${ }^{(24)}$, à inclusão de pacientes com cardiopatias e neuropatias que apresentavam maior idade gestacional e às diferenças no nível socioeconômico entre as populações. Em determinadas situações, em função das condições de renda e moradia, foi necessário prolongar a internação hospitalar para garantir segurança à alta, o que pode ter colaborado para a menor duração do $\mathrm{O}_{2}$ no domicílio.

Neste estudo, a DBP foi a doença mais frequente, confirmando dados da literatura ${ }^{(8,21,23)}$, seguida pela doença do refluxo gastroesofágico. Acredita-se que a elevada incidência de DRGE entre estes pacientes esteja também associada à frequente realização de exames diagnósticos para esta entidade mórbida no serviço durante o período avaliado ${ }^{(25)}$.

A frequência da utilização de $\mathrm{O}_{2}$ domiciliar por $\mathrm{RN}$ de muito baixo peso varia amplamente entre os diferentes centros. São encontrados valores inferiores a 1 até $37 \%$ de uso $^{(26,27)}$. No presente estudo, esta frequência oscilou ao longo do tempo, embora sem significado estatístico. Oscilações na frequência de uso de $\mathrm{O}_{2}$ domiciliar em um mesmo serviço também foram observadas por outros autores ${ }^{(23)}$. Várias diferenças, tanto intra como inter-hospitais, podem colaborar para tais achados e, dentre elas, pode-se considerar o tamanho do hospital e da população de recém-nascidos de muito baixo peso atendida ${ }^{(16,26)}$, receio da equipe de saúde em relação ao impacto e aos custos desta terapêutica para a família ${ }^{(16,24,26)}$, indefinição quanto aos valores de $\mathrm{SaO}_{2}$ indicados para estes pacientes ${ }^{(16,20,24,26,28)}$ e incertezas sobre os benefícios da oxigenoterapia ${ }^{(29,30)}$. A despeito das indefinições, o valor da $\mathrm{SaO}_{2}<90 \%$ é o mais utilizado para o $\mathrm{O}_{2}$ suplementar ${ }^{(16,24,26)}$.

Outro aspecto importante neste estudo é a inexistência de correlação entre a duração da ventilação mecânica e o uso de $\mathrm{O}_{2}$ na internação com a duração da oxigenoterapia 
domiciliar. Suspeitava-se de uma correlação positiva significativa entre estas variáveis, denotando uma maior gravidade da doença de base. Entretanto, são múltiplas as indicações de ventilação mecânica no prematuro, entre elas a sepse e a apneia da prematuridade. Assim, um longo período de ventilação ou uso de $\mathrm{O}_{2}$ intra-hospitar pode não se correlacionar com a dependência prolongada da oxigenoterapia.

Os neonatos com DBP e uso de $\mathrm{O}_{2}$ prolongado também apresentaram outras limitações para a alta, como dificuldade de sucção, utilização de sondas enterais, traqueostomias, além da administração de um grande número de medicações. Desta forma, é essencial que exista uma equipe de enfermagem especializada no preparo dos cuidadores e com competência para avaliar e acompanhar os pacientes após a alta ${ }^{(31)}$. Com este intuito, as mães foram instruídas desde os cuidados básicos de higiene até a fixação de sondas e cateteres e administração correta das medicações, que variaram de uma a 16 doses diárias. Esta etapa também visou a amenizar o sentimento de insegurança dos cuidadores, uma vez que são descritas repercussões sobre a saúde mental das mães ${ }^{(20,22)}$.

A literatura aponta morbidades pulmonares frequentes nos dois primeiros anos de vida dos pacientes em oxigenoterapia domiciliar, com elevadas taxas de reinternações, variando de $45-83 \%{ }^{(32)}$. No presente estudo, $40 \%$ das crianças foram re-hospitalizadas pelo menos uma vez após a alta hospitalar. Não foram obtidas informações recentes e específicas sobre mortalidade após a alta em prematuros utilizando $\mathrm{O}_{2}$ em casa. Dados de pelo menos uma década atrás registravam que a taxa de mortalidade após a alta hospitalar para prematuros com DBP pode variar de 8 a $20 \%{ }^{(33)}$.

\section{Referências bibliográficas}

1. Sociedade Brasileira de Pneumologia e Tisiologia. Oxigenoterapia domiciliar prolongada. J Pneumologia 2000;26:341-50.

2. Palomino MA, Morgues M, Martínez F. Management of infants with chronic lung disease of prematurity in Chile. Early Hum Dev 2005;81:143-9.

3. Kotecha S, Allen J. Oxygen therapy for infants with chronic lung disease. Arch Dis Child Fetal Neonatal Ed 2002;87:F11-4.

4. Mocelin HT, Fischer GB, Ranzi LC, Rosa RD, Philomena MR. Oxigenoterapia domiciliar em crianças: relato de sete anos de experiência. J Pneumologia 2001;27:148-52.

5. Pinney MA, Cotton EK. Home management of bronchopulmonary dysplasia. Pediatrics 1976;58:856-9.

6. Abman SH, Wolfe RR, Accurso FJ, Koops BL, Bowman CM, Wiggins JW Jr. Pulmonary vascular response to oxygen in infants with severe bronchopulmonary dysplasia. Pediatrics 1985;75:80-4.
Devido à escassez de recursos e de suporte do sistema de saúde para o atendimento domiciliar dos pacientes em uso de $\mathrm{O}_{2}$, o programa ofereceu atendimento após a alta somente no ambiente hospitalar, ao contrário de outros relatos ${ }^{(23,32)}$. Também não foram utilizados monitores cardíacos ou oxímetros de pulso no domicílio. Não há evidências de que tal monitoração melhore os resultados da oxigenoterapia domiciliar. No entanto, alguns serviços utilizam este recurso para regular o fluxo de $\mathrm{O}_{2}^{(34)}$ e identificar o deslocamento do cateter $^{(32)}$. Entretanto, os falsos alarmes por artefatos podem atuar como uma fonte de estresse para os cuidadores ${ }^{(34)}$.

Uma limitação deste estudo é o seu caráter retrospectivo, com possibilidade de subnotificação de informações. Assim, não foi possível avaliar com precisão as intercorrências no ambiente domiciliar. Além disso, as condições socioeconômicas foram identificadas para uma pequena amostra da população. Entretanto, os resultados aqui apresentados sugerem que, mesmo com baixas condições socioeconômicas, é possível oferecer oxigenoterapia domiciliar como alternativa à internação hospitalar prolongada. Dadas as limitações apresentadas, é necessário planejar um estudo específico que esclareça melhor as condições socioeconômicas e as possíveis necessidades não supridas no acompanhamento dos familiares, uma vez que pode existir impacto negativo na qualidade de vida das famílias ${ }^{(20,22)}$.

Este estudo demonstrou, por meio da análise dos resultados ao longo de dez anos, que o uso de oxigênio domiciliar é uma alternativa terapêutica factível em nosso meio, que merece mais atenção dos profissionais de saúde e maior investimento das instituições de saúde. Neste contexto, outros estudos são necessários para melhorar a qualidade e segurança desta modalidade terapêutica.

7. Tay-Uyboco JS, Kwiatkowski K, Cates DB, Kavanagh L, Rigatto H. Hypoxic airway constriction in infants of very low birth weight recovering from moderate to severe bronchopulmonary dysplasia. J Pediatr 1989;115:456-9.

8. Gray PH, Rogers Y. Are infants with bronchopulmonary dysplasia at risk for sudden infant death syndrome? Pediatrics 1994;93:774-7.

9. Groothuis JR, Rosenberg AA. Home oxygen promotes weight gain in infants with bronchopulmonary dysplasia. Am J Dis Child 1987;141:992-5.

10. Hudak BB, Allen MC, Hudak ML, Loughlin GM. Home oxygen therapy for chronic lung disease in extremely low- birth- weight infants. Am J Dis Child 1989;143:357-60.

11. Rede Brasileira de Pesquisas Neonatais [homepage on the Internet]. Banco de dados da Rede Brasileira de Pesquisas Neonatais 2007 [cited 2006 jul 15]. Available from: http://www.redeneonatal.fiocruz.br/index. php?option=com_content $\&$ view $=$ article $\&$ id $=6 \& \mid$ temid $=16$ 
12. Greenough A, Alexander J, Burgess S, Chetcuti PA, Cox S, Lenney W et al. High versus restricted use of home oxygen therapy, health care utilization and the cost of care in chronic lung disease infants. Eur J Pediatr 2004;163: 292-6.

13. Committee on Fetus and Newborn. American Academy of Pediatrics. Hospital discharge of the high-risk neonate-proposed guidelines. Pediatrics 1998;102:411-7.

14. Monin P, Vert P. The management of bronchopulmonary dysplasia. Clin Perinatol 1987;4:531-49.

15. Balfour-Lynn IM, Primhak RA, Shaw BN. Home oxygen for children: who, how and when? Thorax 2005;60:76-81.

16. Poets CF. When do infants need additional inspired oxygen? A review of the current literature. Pediatr Pulmonol 1998;26:424-8.

17. Singer L, Martin RJ, Hawkins SW, Benson-Szekely LJ, Yamashita TS, Carlo WA. Oxygen desaturation complicates feeding in infants with bronchopulmonary dysplasia after discharge. Pediatrics 1992;90:380-4.

18. Capurro H, Konichezky S, Fonseca D, Caldeyro-Barcia R. A simplified method for diagnosis of gestational age in the newborn infant. $J$ Pediatr 1978;93:120-2.

19. Ballard JL, Khoury JC, Wedig K, Wang L, Eilers-Walsman, Lipp R. New Ballard Score, expanded to include extremely premature infants. J Pediatr 1991;119:417-23.

20. McLean A, Townsend A, Clark J, Sawyer MG, Baghurst P, Haslam R et al. Quality of life of mothers and families caring for preterm infants requiring home oxygen therapy: a brief report. J Paediatr Child Health 2000;36:440-4.

21. Baraldi E, Carra S, Vencato F, Filippone M, Trevisanuto D, Milanesi O et al. Home oxygen therapy in infants with bronchopulmonary dysplasia a prospective study. Eur J Pediatr 1997;156:878-82.

22. Zanardo V, Freato F. Home oxygen therapy in infants with bronchopulmonary dysplasia: assessment of parental anxiety. Early Hum Dev 2001;65:39-46.
23. Saletti A, Stick S, Doherty D, Simmer K. Home oxygen therapy after preterm birth in Western Australia. J Paediatr Child Health 2004;40:519-23.

24. Ellsbury DL, Acarregui MJ, McGuinnes GA, Eastman DL, Klein JK. Controversy surrounding the use of home oxygen for premature infants with bronchopulmonary dysplasia. J Perinatol 2004;24:36-40.

25. Mezzacappa MA, Collares EF. Prolonged esophageal $\mathrm{pH}$ monitoring utilization in the diagnosis of pathologic reflux in neonates. J Pediatr (Rio J) 1999;75:23743.

26. Peter C, Poets C. Prescription of home oxygen therapy to infants in Germany. Biol Neonate 2001;80:148-51.

27. Fanaroff AA, Stoll BJ, Wright LL, Carlo WA, Ehrenkranz RA, Stark AR et al. Trends in neonatal morbidity and mortality for very low birthweight infants. Am J Obstet Gynecol 2007;196:147.e1-8.

28. Solis A, Harrison G, Shaw BN. Assessing oxygen requirement after discharge in chronic lung disease: a survey of current practice. Eur J Pediatr 2002;161:42830.

29. Halbower AC, McGrath SA. Home oxygen therapy: the jury is still in session. J Perinatol 2004;24:59-61.

30. Lal MK, Sinha S. Oxygen therapy and monitoring newborn infants. J Pediatr Child Health 2004;40:504-5.

31. American Academy of Pediatrics. Hospital discharge of the high-risk neonate. Committee on fetus and newborn. Pediatrics 2008;122:1119-26.

32. Chien YH, Tsao PN, Chou HC, Tang JR, Tsou KI. Rehospitalization of extremely-low-birth-weight infants in first 2 years of life. Early Hum Dev 2002;66:33-40.

33. Moyer-Mileur LJ, Nielson DW, Pfeffer KD, Witte MK, Chapman DL. Eliminating sleep-associated hypoxemia improves growth in infants with bronchopulmonary dysplasia. Pediatrics 1996;98:779-83.

34. Balfour-Lynn IM. Domiciliary oxygen for children. Pediatr Clin North Am 2009;56:275-96. 